Fecha de recepción: marzo 2020 Fecha de aceptación: abril 2020 Versión final: mayo 2020

\section{Tejiendo el Buen Vivir para la (re)conexión con la herencia cultural a través de la práctica textil}

Diana Albarrán González ${ }^{(1)}$

\begin{abstract}
Resumen: El ensayo explora el Buen Vivir en la práctica textil colectiva para la colaboración y ayuda mutua entre grupos de mujeres. Este concepto es una contribución de algunos pueblos originarios de Abya Yala abordando el bienestar colectivo y relación armónica con el entorno natural-cultural. A partir del trabajo colaborativo con un colectivo de tejedoras Mayas de los Altos de Chiapas, se ha identificado que los talleres de co-diseño son un medio de exploración creativa, cultural, visual y textil, creando espacios de (re)conexión social entre mujeres. Por lo tanto, se proponen talleres similares para mujeres migrantes Latinoamericanas para tejer el Buen Vivir desde la transformada mirada identitaria.
\end{abstract}

Palabras clave: Buen Vivir - Práctica Textil - Identidad - (Re)conexión - Cultura - Migración - Aculturación.

[Resúmenes en inglés y portugués en las páginas 80-81]

(1) Doctora en estudios Indígenas por la Auckland University of Technology en Nueva Zelanda. Investigadora en diseño sobre la descolonización del diseño artesanal y el co-diseño, el reconocimiento de formas de diseño indígena y principios del Buen Vivir para mejorar colaboraciones entre artesanos y diseñadores. Cuenta con estudios de Maestría en Diseño, Gestión y Desarrollo de Nuevos Productos de la Universidad Politécnica de Valencia en España, Licenciatura en Diseño Industrial por la Universidad Autónoma de Guadalajara en México, y una estancia técnica en Modern Design and Traditional Craftsmanship en el Kyoto Institute of Technology en Japón. Co-autora del libro Diseño Estratégico: de la estrategia a la gestión del diseño en México publicado por la Editorial Digital del Tecnológico de Monterrey. Tiene más de 15 años de experiencia internacional ejerciendo y enseñando diseño en países como Nueva Zelanda, Singapur, Japón, España y México.

\title{
Prefacio
}

Comienzo este ensayo escribiendo en primera persona como un acto de descolonización y reto a la academia del Norte, para romper el lenguaje impersonal como 'el modo correcto' de escritura y objetividad académica (Mignolo, 2003). Haciendo eco a Gutiérrez Borrero (2015), 
en el Sur(es), los argumentos son personalizados. De igual modo, busco disolver la barrera del individualismo desde el yo para dar pie a la colectividad del nosotros, en dónde mis compañeras de investigación son co-creadoras a través de diálogos vividos, sentidos y tejidos desde la horizontalidad, transformándonos en "yosotros", como es expresado en lengua Maya Tseltal.

\section{Introducción}

Este ensayo discute la 'tejida' investigación para un Buen Vivir junto a mis compañeras tejedoras Mayas Tsotsiles y Tseltales del colectivo Malacate Taller Experimental Textil en los Altos de Chiapas, en el sureste de México. A partir de las exploraciones en talleres de co-diseño sobre el Buen Vivir de los pueblos Tsotsiles y Tseltales, Lekil Kuxlejal, se ha identificado que la estrecha relación entre el trabajo textil de la cotidianidad y el bienestar individual y colectivo, al igual que es un medio para lograr una vida digna y justa.

El Buen Vivir es un concepto-filosofía de vida de algunos pueblos originaria de Abya Yala que aborda el bienestar colectivo y relación armónica con el entorno natural-cultural. A partir de esta premisa, surge la inquietud de explorar como el Buen Vivir nos puede ayudar como mujeres latinoamericanas migrantes creando espacios de (re)conexión como comunidad en diáspora contribuyendo al bienestar individual-colectivo a través de prácticas textiles y creativas.

\section{Posicionalidad(es)}

La posicionalidad, y su intrínseca reflexividad, juegan un papel importante dentro de la investigación decolonial y feminista, usadas como estrategias que se desligan de acercamientos absolutos, a la vez que permiten pensar y crear espacios otros para la generación de nuevos conocimientos (Ali, 2015; Bover, 2013; García Martínez, 2018). Mi papel como investigadora no solo se sitúa, sino se posiciona y "toma su lugar en un medio, crece en él, se desarrolla en él, y también se opone a él” (Greene, 1966, p. 42). En este sentido, es necesario establecer mi postura dentro de los distintos espacios que se desarrolla la investigación y, a su vez, activismo.

Mi posicionalidad se da como diseñadora mexicana en diáspora, una mujer mestiza en proceso de descolonización de sus identidades, una madre migrante activa dentro de la comunidad nativo-latinoamericana en Auckland, Aotearoa Nueva Zelanda. Este territorio con gran presencia Māori, ha tenido influencia no sólo en cómo hacer investigación, sino también en cuestionamientos identitarios sobre el mundo y cosmovisión Indígena. De igual modo, el uso de la teoría decolonial como marco teórico para la descolonización del diseño artesanal textil requiere de maneras de investigación 'muy otras', de prácticas reflexivas en dónde se cuestiona nuestra posición de poder y privilegio académico, e inevitablemente, nuestras distintas identidades. Estos cuestionamientos desde la descolonización han representado transformaciones sobre mi visión del mestizaje en México y Latinoa- 
mérica. Haciendo eco de las palabras de la pensadora Boliviana Silvia Rivera Cusicanqui, descolonizando día a día nuestras subjetividades como mestizos en un proceso para generar conocimiento desde lo cotidiano (Barber, 2019; La Tinta, 2016; Sagárnaga, 2019). El abordar mis identidades durante el trabajo de campo en Chiapas, y de activismo comunitario en Aotearoa, me han permitido tener conocimiento de la visión del Lekil Kuxlejal de mis compañeras tejedoras Mayas, al igual que establecer conexiones con el Buen Vivir como medio de bienestar colectivo con un grupo de mujeres migrantes latinoamericanas.

\section{Buen Vivir, contribuciones para el bienestar colectivo desde Abya Yala}

El Buen Vivir ha ganado reconocimiento como aporte de algunos pueblos originarios para la descolonización y generación de formas otras de vivir, hacer y ser. Se identifica el origen de este concepto desde el Sumaq Kawsay en Quechua y el Suma Qamaña en Aymara como "sistemas de conocimiento, práctica y organización de los pueblos originarios de los Andes de Sudamérica" (Solón, 2017, p. 13), integrándose en las constituciones de Ecuador y Bolivia en 2008 y 2009 respectivamente (Cubillo-Guevara \& Hidalgo-Capitán, 2016; Gutiérrez Borrero, 2015).

El Buen Vivir permite generar alternativas para la construcción de sociedades en armonía en dónde coexisten seres humanos con sus respectivas diversidades culturales y con la naturaleza, en otras palabras un bienestar colectivo (Gudynas \& Acosta, 2011). Estas creencias y principios de vida forman parte de varios pueblos originarios en Abya Yala, con sus respectivas diferencias en denominación y maneras de manifestarse. Algunos equivalentes al Buen Vivir son:

Tabla 1. Buen Vivir de algunos pueblos originarios en Abya Yala

\begin{tabular}{llll}
\hline Nombre & Grupo Indígena & Traducción general & País de origen \\
\hline Küme Mongen & Mapuche & Calidad de Vida & Chile \\
Lekil Kuxlejal & (Maya) Tsotsil y Tseltal & Vida Digna y Justa & México \\
Penker Pujustin & Shuar & Bienestar, Vivir Bien & Ecuador \\
Shiir Wara & Achuar & Bienestar, Vivir Bien & Ecuador \\
Sumak Kawsay & Quechua & Vivir Bien, Buen Vivir & Ecuador \\
Suma Qamaña & Aymara & Vivir Bien, Buen Vivir & Bolivia \\
Teko Kavi, Ñandereko & Guaraní & Vida Armoniosa & Paraguay \\
Utz K'aslemal & (Maya) K'iche y Kaqchikel & Buena Vida & Guatemala \\
Yeknemillis & Nahua & Vida Buena & México
\end{tabular}


Si estas contribuciones para un bienestar colectivo desde distintos países de Latinoamérica bajo el concepto de Buen Vivir están ligadas a nuestras tierras de origen, ¿cómo estos principios para un bienestar colectivo podrían beneficiarnos como miembros de comunidades nativo-latinoamericanas migrantes? ¿el origen indígena del Buen Vivir puede servirnos para conectar con nuestras raíces como práctica descolonizadora? ¿descolonizar nuestra identidad mestiza, especialmente en diáspora, nos permite tener mejores relaciones con nuestros hermanos de pueblos originarios? Estas preguntas surgieron de aprendizajes y reflexiones generadas después del trabajo de campo con mis compañeras tejedoras Mayas Tsotsiles y Tseltales en los Altos de Chiapas, a partir de la exploración colectiva del Lekil Kuxlejal como una vida digna y justa, mismas que han comenzado a explorarse con otras mujeres migrantes latinoamericanas en Nueva Zelanda, punto a desarrollar en la última sección de este artículo.

\section{Mis compañeras de Malacate Taller Experimental Textil}

Malacate Taller Experimental Textil es un colectivo autónomo de mujeres tejedoras y bordadoras Mayas Tsotsiles, Tseltales y Tojolabales de los Altos de Chiapas, en el sureste de México, dedicado al diseño y creación textil desde su cosmovisión y saberes Indígenas desde el 2010 (Malacate Taller Experimental Textil, 2018). Este grupo es reconocido en la región por ser miembros activos dentro de los movimientos para la reivindicación de los diseños y textiles como parte de los derechos de los pueblos originarios y con prácticas éticas dentro del campo del diseño artesanal textil.

En Latinoamérica, el diseño artesanal es un campo en dónde artesanos y diseñadores establecen colaboraciones para el desarrollo de productos artesanales. En el caso de México, el diseño artesanal surge a finales de los años setenta, instaurándose a inicios de los años ochenta (Perez Canovas, 2014). En estos espacios se ha considerado necesaria la intervención del diseño sobre la artesanía, particularmente los textiles, para la creación de piezas artesanales para el consumo fuera de las comunidades Indígenas, adecuándose a mercados con mayor poder adquisitivo (Guzmán Donsel \& García Quintero, 2010; Montalvo de Payes, 2009; Perez Canovas, 2014). De acuerdo con el Fondo Nacional para el Fomento de las Artesanías, los diseñadores artesanales tienen la tarea de ir más allá del enfoque estético de las piezas artesanales buscando el bienestar de las comunidades artesanales. Al mismo tiempo, es necesario que conozcan las demandas y nuevas tendencias del mercado de la moda para establecer estrategias adecuadas para estos mismos (FONART, 2007). Pese a ser un área del diseño establecida, aún existen prácticas que no benefician a las comunidades artesanales Indígenas y vulneran diseño y procesos artesanales, ya que estos no son reconocidos como parte de los derechos de los pueblos originarios como lo establece la declaración sobre los derechos de los pueblos Indígenas de las Naciones Unidas en sus artículos 11 y $31^{2}$.

A diferencia de otros casos en la región, Malacate es de las pocas organizaciones que no cuenta con una diseñadora educada bajo el diseño hegemónico (Eurocentrista, enfocado al consumo), las maestras artesanas-diseñadoras son las que proponen nuevos productos. 
Algunas de las características de los procesos de diseño de Malacate son: 1) generación de nuevos diseños a partir del rescate de técnicas textiles casi desaparecidas, 2) adecuando diseños tradicionales-ceremoniales en nuevas piezas textiles para mercados externos, pero manteniendo la integridad de símbolos sagrados, 3) balanceando las demandas del mercado con sus propias capacidades productivas bajo esquemas de comercio ético.

La comercialización de las piezas textiles artesanales dentro del comercio justo juega un papel importante dentro de los procesos de diseño y producción, por lo que resulta relevante mencionar el modo operativo de Malacate, similar al de otros colectivos textiles independientes en México. Para muchos de estos grupos la autogestión, es decir, el no depender económicamente de fondos de gobierno o de organizaciones no gubernamentales, les permite mantener autonomía e integridad sobre los diseños, tiempos y modos de mercantilización. Por tal motivo, el uso de las redes sociales como Facebook e Instagram ha sido parte fundamental para la comercialización de sus piezas, ya que les permite tener contacto directo con posibles clientes, al igual que brinda una plataforma para compartir información sobre las piezas textiles como procesos de tejido, historias sobre las artesanas y sus contextos comunitarios, intercambios con artesanas de otros estados, colaboraciones con colectivos textiles y participación en mercados fuera del estado. Estos modos 'alternativos' de operación les permite conservar prácticas de diseño y producción otras, respetando lo que ellas consideran justo dentro del comercio del diseño artesanal textil sin anteponer las demandas de un mercado capitalista. En este sentido, la comercialización de sus piezas artesanales textiles funciona en una lógica distinta a la del capital, operando bajo economía social (Caracciolo, 2017; Oulhaj \& Gallejos, n.d.). Este modelo está alineado con sus valores como colectivo independiente en dónde el objetivo no es la venta, sino un medio para alcanzar una vida digna y justa, Lekil Kuxlejal.

\section{Explorando el Lekil Kuxlejal a través del co-diseño desde la descolonización}

El Lekil Kuxlejal, considerado el Buen Vivir de los pueblos Mayas Tsotsiles y Tseltales de Chiapas, se traduce a grandes rasgos como Lekil, bueno, y Kuxlejal, vida. También reconocido como vida digna y justa, es un modo de ser, actuar, existir en armonía entre humanos y naturaleza, reconociendo y respetando la autonomía de todos los seres vivos y los pueblos. (Prage, 2015; Schlittler, 2012). Para estos grupos Indígenas, el jolobil (tejido) es parte importante de la vida cotidiana y el bienestar colectivo, relacionado con el Lekil Kuxlejal, como lo explica el sociólogo Tseltal López Intzin (n.d.):

Tanto el hilo, los palitos, como el kuchupat, que le permitirá la tejedora o tejedor estar dentro del telar de cintura también forman una unidad, que entre sí comulgan para formar un cuerpo, una comunidad [...] Las manos danzan y acarician cada parte del telar. Al final de la jornada, se presenta el trabajo concluido. Hay armonía porque el corazón se ríe, la comunidad se viste de flor en cada ocasión especial [...] El tejido, entonces, como un trabajo concluido, es la realización máxima de la tejedora portado y presumido por los miembros 
de la comunidad. Embriaga su corazón de risa, ya stse'e yo'tan, ak'ol k'inal ya $y a ’$, siente que el medio ambiente, el universo se está dando para ella (p. 6, 7).

Esta relación entre la artesanía textil en el diario hacer, parte de una vida digna y justa, nos ha servido de base para la exploración del Lekil Kuxlejal a través de talleres de co-diseño en dónde el lenguaje visual-sensorial rompen las barreras de la lengua hablada. La propuesta, entonces, sugiere el uso del Lekil Kuxlejal como marco de referencia para la descolonización del diseño artesanal textil en la región de los Altos de Chiapas y la creación de guías para colaboraciones éticas y respetuosas desde la perspectiva de las tejedoras Mayas, y no desde actores externos.

El co-diseño como acción colaborativa, permite romper las prácticas hegemónicas-individualistas del diseño al igual que la jerarquía entre los participantes. Similar al diseño participativo, este acercamiento tiene como objetivo replantear el sentido de expertise y las relaciones de poder (Huybrechts, Benesch, \& Geib, 2017, p. 149), en dónde el rol del diseñador consiste en facilitar el trabajo colectivo y los participantes son expertos de sus propias experiencias y realidades (Sanders \& Stappers, 2014). Por tal motivo, se organizaron dos talleres de co-diseño enfocados en la exploración visual-sensorial-afectiva del Lekil Kuxlejal, teniendo como base la reciprocidad y el respeto a la autonomía del grupo y la comunidad (Akama, Hagen, \& Whaanga-Schollum, 2019), ideas alineadas con prácticas descolonizadoras (ver Figura 1).

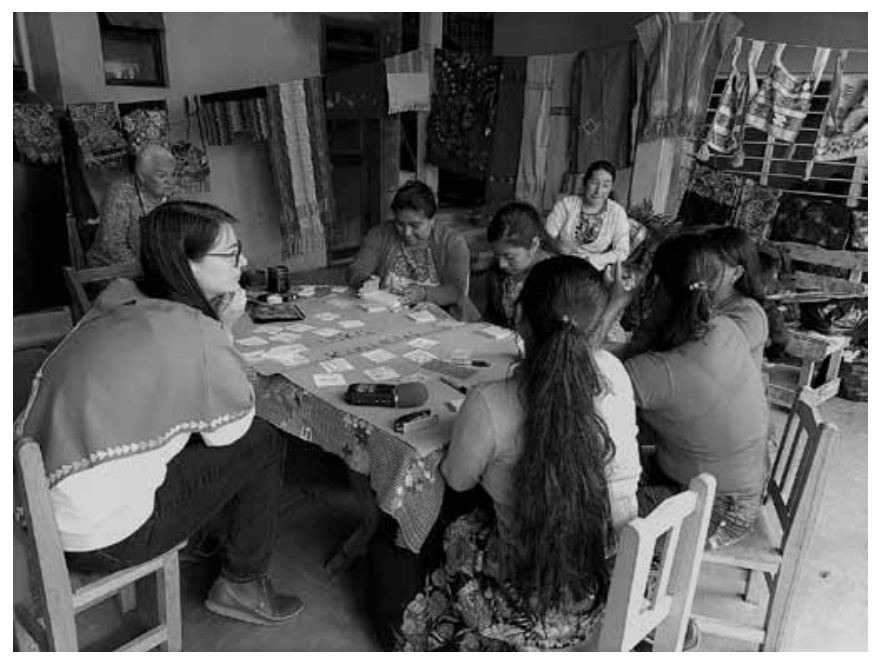

Figura 1. 
Las experiencias vividas con mis compañeras de Malacate han impactado mi manera de ver, actuar y existir en los distintos roles y espacios en los que habito, mis distintas posiciones. Por consecuencia, surgen cuestionamientos de cómo estos aprendizajes pueden beneficiar otras comunidades de mujeres, especialmente en situaciones vulnerables como en situación de migración (Rosales Mendoza, 2017), misma que he vivido en distintos países a lo largo de mi vida. Por tal motivo, surge la inquietud de actuar más allá de espacios académicos a través de investigación y acción activa en el contexto en donde resido, al igual como lo han hecho otras investigadoras (por ejemplo Ali, 2015; De la Piedra \& Méndez, 2018).

\section{Migración e identidad, entre asimilación y transformación}

La migración es un fenómeno presente en la historia de la humanidad. De acuerdo con los datos de la Organización Internacional para las Migraciones (2013), existen 232 millones de migrantes internacionales, dentro de los cuáles 110 millones son mujeres, estableciendo un $49 \%$ del total. Como consecuencia de la experiencia migratoria, entramos en contacto con una cultura nueva en dónde existe adopción de valores y prácticas culturales distintas a la de origen en un proceso llamado aculturación (Berry, 2005; Cole, 2019). Frecuentemente somos cuestionadas sobre nuestros orígenes en el nuevo espacio de residencia, especialmente cuando somos visiblemente distintas a la población de origen, generando cuestionamiento sobre nuestras identidades.

El ser una migrante y ocupar un espacio como 'otro' resulta una experiencia que genera tensión, conflicto y sentimientos de dislocación impactando las percepciones sobre nosotras mismas (Martínez Azaro, 2020). En algunos casos, estas tensiones identitarias generan procesos de asimilación en dónde individuos o grupos de personas adoptan la nueva cultura reemplazando casi completamente la cultura de origen. Este fenómeno es común dentro de las poblaciones migrantes buscando mezclarse con el existente tejido social y no ser reconocidos como extranjeros. Dentro de los procesos de aculturación es inevitable que la cultura de origen sea transformada en el proceso de adaptación en el nuevo territorio, pero es importante mantener las raíces culturales del lugar de origen para nuestro bienestar, especialmente en las primeras etapas de reasentamiento. En las sociedades multiculturales, especialmente dentro de comunidades migrantes minoritarias, es común que sus miembros mantengan su cultura original a la vez que se adaptan a la nueva cultura en un proceso llamado integración (Berry, 2005; Cole, 2019). Si la integración permite adoptar aspectos de la cultura nueva a la vez que nos ayuda a readaptar la cultura de origen, surgen nuevas preguntas como: ¿es posible establecer conexiones más significativas con nuestro lugar de origen como migrante? ¿cómo influye la cultura local en nuestras identidades? ¿la posición de 'otredad' provoca buscar reconectar con nuestra cultura originaria? En el caso particular que el país de acogida tenga una reconocida y fuerte población Indígena, ¿el reconocimiento de la identidad nativa local influye en replanteamientos de nuestra identidad mestiza (o criolla) como latinoamericanas? 
Investigadores latinoamericanos en el exterior han experimentado transformaciones identitarias desde su nueva posición como migrante con distintos resultados. Martínez Azaro (2020) explica como sus experiencias como migrante latinoamericana transformaron su autopercepción y el modo de hacer trabajo creativo. En relación con la identidad mestiza, varios académicos manifestaron tensiones y transformaciones buscando (re)conocer y (re)conectar con su origen Indígena como refiere la siguiente cita:

Comenzó a aflorar en mí esa tensión interior que vive el mestizo, provocada por la contienda entre su raíz indígena y su raíz europea [...] Desde el terreno del colonizador, he sentido y conocido mi Sur como probablemente no lo habría hecho permaneciendo en el terreno del colonizado (Marín-Burgos \& Enríquez Paz y Puente, 2015, p. 181).

Otras investigadoras han (re)conectado con sus raíces Indígenas a partir de relacionarse con grupo nativos (Albarran Gonzalez, 2019; Benton Zavala, 2018) o a través del trabajo y exploración del textil vernáculo en el nuevo asentamiento (Sahagún Sánchez, 2015). A partir de ejemplos como estos, surge la inquietud de explorar la creación textil como medio de (re)conexión nuestras raíces nativo-latino americanas como mujeres migrantes en Aotearoa.

\section{Experiencias tejidas entre la investigación de campo en México y como miembro de la comunidad migrante Latinoamericana en Aotearoa Nueva Zelanda}

Como mencioné anteriormente, considero relevante llevar los aprendizajes y resultados de la investigación a contextos no académicos, a espacios en dónde otras mujeres puedan ser beneficiadas a nivel individual y como comunidad. En este sentido, este estudio puede considerarse investigación activista que se realiza desde la teoría para ser llevada a la práctica (De la Piedra \& Méndez, 2018). Por tal motivo, se busca la aplicación de los aprendizajes de la investigación de campo junto con mis compañeras de Malacate, y el Buen Vivir como convivencia armónica y bienestar colectivo, puedan ser explorados y desarrollados junto con mujeres migrantes nativo-latinoamericanas en Aotearoa para la (re)conexión con la herencia cultural a través de la práctica textil.

El primer acercamiento piloto se realizó dentro del marco del 3er. Foro Femenino "Creciendo en Sororidad" organizado por ALAC Inc [Aotearoa Latin American Community] (ALAC Inc, 2019). ALAC Inc es una organización no gubernamental que lleva 25 años apoyando a la comunidad latinoamericana refugiada y migrante en Aotearoa. Este evento de un día se centró en (re)conectar a mujeres nativo-latinoamericanas a través de charlas y pequeños talleres, mismo al que fui invitada como ponente.

Mi ponencia-taller tuvo como objetivo dos aspectos principalmente: 1) explicar a grandes rasgos el Buen Vivir y algunos de sus principios como un relevante aporte de algunos pueblos originarios, 2) usar ejercicios simbólicos textiles para reflexionar sobre nuestras 
conexiones como mujeres y con nuestras ancestras. A través de la co-creación de un simbólico aro de bordado, las participantes 'tejieron' deseos colectivos como mujeres migrantes nativo-latinoamericanas para un Buen Vivir en Aotearoa (ver Figura 2). Cabe destacar que este fue un ejercicio exploratorio con limitaciones de tiempo imposibilitando su realización como taller de co-diseño. Sin embargo, este primer acercamiento ha servido como base para el desarrollo de futuros talleres.

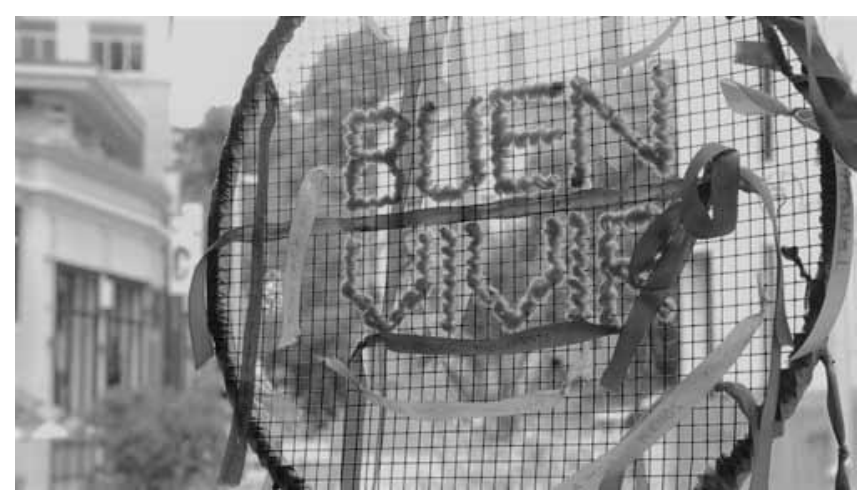

Figura 2.

\section{Reflexiones}

El Buen Vivir como contribución de algunos pueblos originarios de Abya Yala para un bienestar colectivo ha ido posicionándose en distintos espacios como la academia, la política, y los movimientos sociales dentro de nuestros territorios en América Latina. Esta filosofía hacia una vida buena puede ser utilizada dentro de comunidades en diáspora para la búsqueda del bienestar individual y colectivo. Por otro lado, los conocimientos adquiridos a partir de trabajos de investigación académica sobre el Buen Vivir pueden ser explorados, readaptados y llevados a la práctica para el beneficio de comunidades migrantes, especialmente en situación vulnerable. De igual modo, se ha identificado que el trabajo textil se relaciona con el bienestar individual y comunitario, y pueden ser usados como medios de exploración, reflexión y (re)conexión. Por tal motivo, se proponen talleres en dónde grupos de nativo-latinoamericanas migrantes puedan generar espacios de (re)conexión como mujeres, comunidad y con la herencia cultural originaria para la transformación positiva de la autopercepción y transformación identitaria como consecuencia de procesos de aculturación como migrantes. 


\section{Notas}

1. Abya Yala es el nombre que el pueblo Guna de Colombia y Panamá (también conocido como Kuna) usa para nombrar al Continente Americano. El término significa Tierra Madura, Tierra Viva o Tierra en Florecimiento.

2. Artículo 11

1. Los pueblos indígenas tienen derecho a practicar y revitalizar sus tradiciones y costumbres culturales. Ello incluye el derecho a mantener, proteger y desarrollar las manifestaciones pasadas, presentes y futuras de sus culturas, como lugares arqueológicos e históricos, objetos, diseños, ceremonias, tecnologías, artes visuales e interpretativas y literaturas.

Artículo 31

1. Los pueblos indígenas tienen derecho a mantener, controlar, proteger y desarrollar su patrimonio cultural, sus conocimientos tradicionales, sus expresiones culturales tradicionales y las manifestaciones de sus ciencias, tecnologías y culturas, comprendidos los recursos humanos y genéticos, las semillas, las medicinas, el conocimiento de las propiedades de la fauna y la flora, las tradiciones orales, las literaturas, los diseños, los deportes y juegos tradicionales, y las artes visuales e interpretativas. También tienen derecho a mantener, controlar, proteger y desarrollar su propiedad intelectual de dicho patrimonio cultural, sus conocimientos tradicionales y sus expresiones culturales tradicionales.

\section{Bibliografía}

Akama, Y.; Hagen, P. \& Whaanga-Schollum, D. (2019). Respectful, Reciprocal, and Relational Co-designing with Indigenous People. Design and Culture, 1(0), qa. https://doi.org/10. 1080/17547075.2019.1571306

ALAC Inc. (2019). Foro Femenino 2019 “Creciendo en Sororidad.” [Video file]. Retrieved from https://www.youtube.com/watch?v=Kj9kARBYfW8

Albarran Gonzalez, D. (2019, February 20). "When we forget": I am Indigenous and I didn't know - K.I.N. Knowledge in Indigenous Networks. [Web log post]. Retrieved August 28, 2019, from https://indigenousknowledgenetwork.net/2019/02/20/when-we-forgeti-am-indigenous-and-i-didnt-know/

Ali, R. (2015). Rethinking representation: negotiating positionality, power and space in the field. Gender, Place and Culture, 22(6), 783-800. https://doi.org/10.1080/096636 9X.2014.917278

Barber, K. (2019, February 17). Feminismo poscolonial | Silvia Rivera Cusicanqui: "Tenemos que producir pensamiento a partir de lo cotidiano." El Salto. Retrieved from https:// www.elsaltodiario.com/feminismo-poscolonial/silvia-rivera-cusicanqui-producirpensamiento-cotidiano-pensamiento-indigena

Benton Zavala, A. M. (2018). 'Shifting the Landscape', Indigenous Immersion and Bilingual Education in Mexico : Tosepan Kalnemachtiloyan. (Doctoral dissertation) The University of Auckland. 
Berry, J. W. (2005). Acculturation: Living successfully in two cultures. International Journal of Intercultural Relations, 29(6), 697-712.

Bover, A. (2013). Herramientas de reflexividad y posicionalidad para promover la coherencia teórico-metodológica al inicio de una investigación cualitativa. Enfermería Clínica, 23(1), 33-37.

Caracciolo, M. (2017). Economía social y solidaria en un escenario neoliberal: algunos retos $y$ perspectivas.

Cole, N. L. (2019). Understanding Acculturation and Why It Happens. Retrieved October 1, 2019, from https://www.thoughtco.com/acculturation-definition-3026039

Cubillo-Guevara, A. P., \& Hidalgo-Capitán, A. L. (2016). El sumak kawsay genuino como fenómeno social amazónico ecuatoriano. OBETS. Revista de Ciencias Sociales, 10(2), 301-333. https://doi.org/10.14198/obets2015.10.2.02

De la Piedra, M. T., \& Méndez, Z. Y. (2018). Reflexiones sobre la complejidad del quehacer antropológico, nuestra posicionalidad, y subjetividades en la frontera (EEUU-México). Anthropologica, 36(41), 9-34. https://doi.org/10.18800/anthropologica.201802.001

FONART. (2007). Manual de Diseño y Desarrollo de Productos Artesanales.

García Martínez, E. (2018). Espacios diferentes e interculturalidad. Posición, pensamiento y posicionalidad otra sobre la Universidad Intercultural del Estado de México. Revista CoPaLA, 6(3), 151-165.

Greene, M. (1966). El concepto de posicionalidad. Introducción a la filosofia de Helmuth Plessner. Convivium, (22), 39-61.

Gudynas, E. \& Acosta, A. (2011). El buen vivir o la disolución de la idea del progreso. La Medición Del Progreso y Del Bienestar: Propuestas Desde América Latina. Mexico City: Foro Consultivo Científico y Tecnológico.

Gutiérrez Borrero, A. (2015). Resurgimientos: sures como diseños y diseños otros. Nomadas, 43, 113-129.

Guzmán Donsel, A. M. \& García Quintero, F. (2010).Diseño, artesanía e identidad. Experiencias académicas locales de Diseño Artesanal en Colombia y El Salvador. Diseño, Artesanía e Identidades (Primera Ed). Popayán: Ediciones Axis Mundi.

Huybrechts, L.; Benesch, H. \& Geib, J. (2017). Institutioning: Participatory Design, CoDesign and the public realm. CoDesign, 13(3), 148-159. https://doi.org/10.1080/15710 882.2017.1355006

La Tinta. (2016, September 15). Seguir mirando a Europa es apostar por un suicidio colectivo. La Tinta. Retrieved from http://iberoamericasocial.com/seguir-mirando-europaapostar-suicidio-colectivo/

López Intzín, J. (n.d.). Ich'el ta muk': la trama en la construcción mutua y equitativa del Lekil kuxlejal (vida plena-digna)., 1-20.

Malacate Taller Experimental Textil. (2018). Nuestro camino, nuestra lucha.

Marín-Burgos, V. \& Enríquez Paz y Puente, Ó. R. (2015). Autoetnografías de descolonización: de cómo dos investigadores latinoamericanos nos comenzamos a descolonizar en el Norte global. Migración y Desarrollo, 13(25), 175-189.

Martínez Azaro, V. (2020). Empatía y Diseño en un contexto de inmigración. en Cuaderno 78 (pp. 29-37). Centro de Estudios en Diseño y Comunicación. 
Migraciones, O. I. para las. (2013). Informe sobre las migraciones en el mundo. Ginebra. Retrieved from http://www.iom.int/

Montalvo de Payes, M. E. (2009). La carrera de Diseño Artesanal y Diseño del Producto Artesanal, 1-7.

Oulhaj, L., \& Gallejos, X. (n.d.).Visibilizando nexos entre la migración y la economía social y solidaria con perspectiva de género.

Perez Canovas, K. (2014). La transformación de la artesanía textil a través de su mercantilización entre diseñadores y tejedoras en los Altos de Chiapas (Master Thesis). Universidad Nacional Autónoma de México.

Prage, L. (2015). Lekil Kuxlejal -an alternative to development? (Master Thesis). Lund University.

Rosales Mendoza, A. L. (2017). “Toda la creencia está allá”. Linderos interculturales, espacios y derechos en migrantes agrícolas temporales de la península de Yucatán en Quebec. Estudios de Cultura Maya, 48, 193-222. https://doi.org/10.19130/iifl.ecm.2016.48.754

Sagárnaga, R. (2019). “Descolonizarme es un proceso de todos los días”: Silvia Rivera Desinformémonos. Desinformémonos. Periodismo de Abajo. Retrieved from https:// desinformemonos.org/descolonizarme-es-un-proceso-de-todos-los-dias-silvia-rivera/

Sahagún Sánchez, V. (2015). Weaving Mestiza Geographies: An A/r/tographic Allegory on Cultural Identity Through the Lens of Vernacular Textile Traditions, (Doctoral dissertation). Concordia University.

Sanders, E. B. N. \& Stappers, P. J. (2014). Probes, toolkits and prototypes: Three approaches to making in codesigning. CoDesign, 10(1). https://doi.org/10.1080/15710882.2014.888183

Schlittler, J. (2012). ¿Lekil Kuxlejal como horizonte de lucha? Una reflexión colectiva sobre la autonomía en Chiapas. (Master Thesis) Centro de Investigaciones y Estudios Superiores en Antropología Social.

Solón, P. (2017). Alternativas Sistémicas (1era. Edic). La Paz, Bolivia: Fundación Solo, Attac France, Focus on the Global South.

Abstract: The essay explores Buen Vivir in collective textile practices for collaboration and mutual support among women. This concept is a contribution from some Indigenous peoples from Abya Yala addressing collective well-being and harmonious relations with natural-cultural environments. Through the experiences with the Mayan weavers collective, it has been identified that co-design workshops are a medium for creative, cultural, visual and textile exploration creating spaces for social (re)connection among women Therefore, similar workshops are proposed for Latin American migrant women to weave Buen Vivir from the transformed identity perspective.

Keywords: Buen Vivir - Textile Practice - Identity - (Re)connection - Cultural - Migration - Acculturation. 
Resumo: O artigo explora o Bom Viver na prática têxtil coletiva para a colaboração e ajuda mútua entre grupos de mulheres. Este conceito é uma contribuição dos povos originários de Latino-América que aborda o bem estar coletivo e a relação harmônica com o entorno natural e cultural. Com as experiências com o coletivo de tecelãs Maya, Malacata Oficina Experimental, foi identificado que as oficinas de design colaborativo são um meio de exploração visual e têxtil criando espaços de (re) conexão social e fortalecimento de relações interculturais. É proposto que estas oficinas para mulheres migrantes vão tecendo o Bom Viver a partir do olhar identitário transformado.

Palavras chave: Bom Viver - Prática Têxtil - Identidade - (Re) Conexão - Cultura - Migração - Aculturação.

[Las traducciones de los abstracts fueron supervisadas por el autor de cada artículo] 